\title{
MECANISMOS DE REMOÇÃO DE CROMO(VI) DO SOLO PELA INTERAÇÃO ENTRE MATÉRIA ORGÂNICA E FERRO(III)
}

\author{
Vanessa MARONEZI \\ Marília Mayumi Augusto dos SANTOS \\ Danielle Bittencourt FARIA \\ Maria Isabel Garcia ROSA \\ Mirian Chieko SHINZATO
}

\begin{abstract}
RESUMO
A contaminação do solo e águas por cromo $(\mathrm{Cr})$ tem ocorrido frequentemente em áreas industriais e em suas proximidades, muitas vezes devido a acidentes e, também, ao mau gerenciamento de seus resíduos. O cromo na forma hexavalente $(\mathrm{Cr}(\mathrm{VI}))$ é extremamente tóxico e cancerígeno, enquanto na forma trivalente $(\mathrm{Cr}(\mathrm{III}))$ e em níveis traço é considerado benéfico aos seres humanos e animais. O presente estudo apresenta uma revisão sobre os principais mecanismos relacionados à interação do $\mathrm{Cr}(\mathrm{VI}) \mathrm{com}$ alguns componentes do solo, quando associado a um evento de contaminação do meio. Observa-se que os principais agentes responsáveis pela remoção do $\operatorname{Cr}(\mathrm{VI})$ do meio são aqueles capazes de reduzi-lo à forma trivalente (Cr(III)), como a fração orgânica do solo e o $\mathrm{Fe}(\mathrm{II})$. No entanto, minerais e compostos ricos em $\mathrm{Fe}$ (III) também podem auxiliar na redução do $\mathrm{Cr}(\mathrm{VI})$. Nesse caso, o Fe(III) pode ser reduzido pelo carbono orgânico à forma divalente que, por sua vez, reduz o cromo à forma trivalente, imobilizando-o no solo. Para que isso ocorra, além da presença de matéria orgânica, o pH ácido do meio também auxilia nesse processo - cujas condições são comumente encontradas em solos tropicais, como o Latossolo Vermelho. Portanto, entender como o $\mathrm{Cr}(\mathrm{VI})$ reage com os principais componentes do solo é muito importante para auxiliar nos processos de remediação de áreas contaminadas.
\end{abstract}

Palavras-chave: Cromo(VI); Contaminação; Solo; Fe(III); Oxirredução; Matéria-orgânica.

\section{ABSTRACT}

REMOVAL MECHANISMS OF CHROMIUM(VI) FROM THE SOIL BY INTERACTION BETWEEN ORGANIC MATTER AND IRON(III). Soil and water contamination by chromium $(\mathrm{Cr})$ has occurred frequently in industrial areas and its vicinity, due to accidents and poor residue management. Chromium in hexavalent form $(\mathrm{Cr}(\mathrm{VI}))$ is highly toxic and carcinogenic, whereas chromium in trivalent form $(\mathrm{Cr}(\mathrm{III}))$ and trace levels of $\mathrm{Cr}$ are considered beneficial for humans and animals. This study presents a review of the main mechanisms related to the interaction of $\mathrm{Cr}$ with soil components, when associated with an environmental contamination event. The main agents responsible for the immobilization of $\mathrm{Cr}(\mathrm{VI})$ are those capable of reducing $\mathrm{Cr}(\mathrm{VI})$ to $\mathrm{Cr}(\mathrm{III})$, such as soil organic fraction and $\mathrm{Fe}(\mathrm{II})$. However, the presence of $\mathrm{Fe}(\mathrm{III})$ minerals/compounds may also aid in the reduction of $\mathrm{Cr}(\mathrm{VI})$. In this case, $\mathrm{Fe}(\mathrm{III})$ can be reduced by organic matter to the divalent form, which, in turn, reduces chromium(VI) to the trivalent form, immobilizing it in the soil. For this to occur, in addition to the presence of organic matter, an acid medium also favors this process - such conditions are typical of tropical soils, such as Oxisol. Therefore, understanding how $\mathrm{Cr}(\mathrm{VI})$ reacts 
with the main components of soil is very important for the remediation of contaminated areas.

Keywords: Chromium(VI); Contamination; Soil; Iron(III); Oxidation-reduction; Organic matter.

\section{INTRODUÇÃO}

$\mathrm{O}$ cromo $(\mathrm{Cr})$ é considerado o $21^{\circ}$ elemento mais abundante da crosta terrestre, com cerca de 122 ppm (MOHAN \& PITTMAN 2006), e o $6^{\circ} \mathrm{em}$ sua escala de metais de transição (GOMES 2017). Sua forma metálica é obtida mediante o processamento da cromita $\left(\mathrm{FeO} . \mathrm{Cr}_{2} \mathrm{O}_{3}\right)$, o mineral com maior aproveitamento econômico (SAMPAIO et al. 2008). Por apresentar resistência à oxidação, desgaste e atrito, este metal é utilizado na fabricação de ligas metálicas, construção civil e indústria química nas áreas de pigmentação, galvanoplastia e curtimento de couro, por exemplo (SAMPAIO et al. 2008, ATSDR 2012, CETESB 2017).

Como todo metal de transição, o cromo possui diferentes estágios de oxidação que vão de $\mathrm{Cr}(\mathrm{II})$ a $\mathrm{Cr}(\mathrm{VI})$, sendo as espécies (III) e (VI) mais comuns no ambiente (KIMBROUGH et al. 1999, SREERAM \& RAMASAMI 2001). Assim sendo, a especiação do cromo é influenciada principalmente pelo potencial redox do meio, ou seja, pelo Eh (condições redutoras ou oxidantes) e pelo $\mathrm{pH}$ (condições ácidas ou básicas) (FRANCO 2008).

As formas de $\mathrm{Cr}(\mathrm{III})$ e (VI) são distintas quanto à mobilidade, toxicidade e características físico-químicas. Em geral, a espécie trivalente do cromo pode ser encontrada naturalmente na forma de minerais e sob ambientes redutores (Eh baixo). É relativamente estável e apresenta uma baixa solubilidade em água sendo, consequentemente, pouco móvel (STANIN \& PIRNIE 2004, CAO \& ZHANG 2006). As espécies que predominam estão sob a forma de $\mathrm{Cr}^{3+}, \mathrm{Cr}(\mathrm{OH})^{2+}, \mathrm{Cr}(\mathrm{OH})_{2}{ }^{+}, \mathrm{Cr}(\mathrm{OH})_{3}{ }^{0}$ (PALMER \& WITTBRODT 1991). A fração solúvel pode ser facilmente adsorvida pelos constituintes do solo e, em concentrações-traço, é até mesmo considerada benéfica aos seres humanos e animais (ATSDR 2012).

As espécies de cromo hexavalente, por sua vez, são altamente tóxicas e carcinogênicas e ocorrem em condições oxidantes (Eh alto) sendo, na maioria dos casos, de origem antrópica. Apresentam alta reatividade e solubilidade em água, podendo ser facilmente transportadas através do solo e atingir as águas subterrâneas (STANIN \& PIRNIE 2004, CAO \& ZHANG 2006, SANTOS et al. 2018). Nesse caso, os íons de cromo que prevalecem na forma hexavalente encontram-se na forma de ânions, como $\mathrm{HCrO}_{4}^{-}, \mathrm{CrO}_{4}^{2-} \mathrm{e} \mathrm{Cr}_{2} \mathrm{O}_{7}^{2-}$ (RAI et al. 1989, SPARKS 2003, CAO \& ZHANG 2006).

Tendo em vista as peculiaridades da especiação do cromo, é necessário reconhecê-las na fonte contaminada, para assim, melhor avaliar o seu grau de toxicidade e auxiliar na escolha de tratamentos mais adequados na área contaminada. Apesar de existirem várias técnicas para tratamento de efluentes contendo $\mathrm{Cr}(\mathrm{VI})$, é comum encontrar ambientes contaminados por esse metal devido a acidentes e/ou mesmo descarte irregular de resíduos e efluentes industriais no solo e águas (SHAHID et al. 2017). Uma vez no ambiente, o $\mathrm{Cr}(\mathrm{VI})$ pode sofrer redução por agentes como a matéria orgânica dissolvida, $\mathrm{Fe}$ (II) e comunidade bacteriana (XIAO et al. 2012).

Entretanto, sob condições tropicais, é muito mais comum encontrar Fe(III) que Fe(II) nos constituintes dos solos. Nesse contexto, cabe ressaltar que há poucos estudos focando nos mecanismos de interação envolvendo compostos com $\mathrm{Fe}$ (III) na remoção de $\mathrm{Cr}(\mathrm{VI})$ de solos tropicais. Desta forma, este trabalho tem por objetivo apresentar as principais interações entre o $\mathrm{Cr}(\mathrm{VI})$ e os compostos com $\mathrm{Fe}(\mathrm{III})$ e matéria orgânica, a fim de aumentar a compreensão dos mecanismos atuantes em solos ricos nesses componentes.

\section{CROMO E MEIO AMBIENTE}

Para controlar os efeitos negativos do cromo no ambiente, a legislação ambiental limitou suas concentrações tanto nas emissões de efluentes industriais, quanto na composição de águas e solos. Assim sendo, segundo a Resolução ${ }^{\circ} 430$ do CONAMA (2011) o valor máximo permitido de lançamento de $\mathrm{Cr}(\mathrm{VI})$ em água é de $0,1 \mathrm{mg} \mathrm{L}^{-1}$ e de $1,0 \mathrm{mg} \mathrm{L}^{-1}$ para $\mathrm{Cr}(\mathrm{III})$. Para as águas superficiais classes 1,2 e 3 (que podem ser usadas ao abastecimento para consumo humano) a Resolução $\mathrm{n}^{\circ}$ 357 do CONAMA (2005) estabeleceu o limite máximo de $0,05 \mathrm{mg} \mathrm{L}^{-1}$ de cromo total. Nos Estados Unidos esse limite é um pouco superior, onde o valor máximo de Cr total permitido é de $0,1 \mathrm{mg} \mathrm{L}^{-1}$ 
nas águas potáveis (USEPA 2017). Para as águas subterrâneas, por sua vez, o valor de intervenção para cromo total é de $50 \mu \mathrm{g} \mathrm{L}{ }^{-1}$ (CETESB 2016).

Quanto às concentrações limites de $\mathrm{Cr}(\mathrm{VI})$ em solos, esses valores variam de acordo com o seu uso, ou seja, se agrícola $\left(0,4 \mathrm{mg} \mathrm{kg}^{-1}\right)$, residencial $\left(3,2 \mathrm{mg} \mathrm{kg}^{-1}\right)$ ou industrial $\left(10 \mathrm{mg} \mathrm{kg}^{-1}\right)$ (CETESB 2016). Quando se considera a concentração de cromo total, esses valores mudam para $150 \mathrm{mg}$ $\mathrm{kg}^{-1}, 300 \mathrm{mg} \mathrm{kg}^{-1}$ e $400 \mathrm{mg} \mathrm{kg}^{-1}$, respectivamente (CETESB 2016).

Portanto, é muito importante que os efluentes industriais contendo $\mathrm{Cr}(\mathrm{VI})$ sejam devidamente tratados, a fim de não contaminarem as fontes de água e o solo, evitando-se, dessa forma, trazer sérios riscos à saúde da população, assim como a fauna e flora local.

Por outro lado, muitos estudos têm revelado que as próprias condições do meio podem ou não favorecer o equilíbrio entre o elemento e os constituintes do solo. Em estudo realizado em um corpo hídrico distante a $30 \mathrm{~m}$ do local de descarte de um lodo galvânico, PUGAS (2007) verificou que não houve contaminação, provavelmente devido à retenção promovida pelas propriedades do solo. FRANCO (2008) também observou em um ambiente contaminado por cromo que alguns processos físicos e químicos influenciaram em seu transporte, composição e forma.

Dessa forma, a Companhia Ambiental do Estado de São Paulo (CETESB 2012) considera o solo como um atenuador da poluição, por possuir capacidade de depuração. No entanto, dependendo das características do solo e da natureza e concentração do contaminante, esse papel pode diminuir, ou mesmo, não fazer efeito (CETESB 2013).

Diante da importância de entender como algumas propriedades do solo podem influenciar no comportamento cinético dos metais (mais especificamente do cromo), serão detalhados a seguir alguns processos, como de sorção (absorção/adsorção), complexação, oxirredução e precipitação/ dissolução.

\subsection{Sorção}

A sorção envolve dois principais mecanismos: a absorção e a adsorção. A absorção é um processo no qual íons, moléculas ou átomos das fases líquida, sólida ou gasosa difundem-se, ou seja, entram e são incorporadas por outra fase (líquida ou sólida) (ESSINGTON 2005). A adsorção, por sua vez, ocorre quando partículas orgânicas ou inorgânicas aderem por ligação molecular ou iônica na superfície de outra substância sólida (SPOSITO 2008).

Dentre os processos de sorção, a adsorção será mais detalhada aqui por ser um dos principais mecanismos que envolve a retenção por interações físicas e químicas de substâncias orgânicas e inorgânicas pelos constituintes dos solos (SPARKS 2003, ESSINGTON 2005). Na adsorção física, a ligação do adsorvato (substância removida) à superfície do adsorvente (material que irá adsorver) é atribuída a forças de Van der Waals, as quais são similares às forças de coesão entre moléculas; enquanto na adsorção química, tem-se uma ligação mais forte derivada de trocas iônicas (SPARKS 2003, ATKINS \& JONES 2012).

Tanto o $\mathrm{Cr}(\mathrm{VI})$ quanto o $\mathrm{Cr}(\mathrm{III})$ podem ser adsorvidos por diversos materiais em solos, águas superficiais ou subterrâneas. Minerais com grupos estruturais de hidroxila $\left(\mathrm{OH}^{-}\right)$- como a caulinita $\left(\mathrm{Si}_{2} \mathrm{Al}_{2} \mathrm{O}_{5}(\mathrm{OH})_{4}\right)$, goethita $(\mathrm{FeO}(\mathrm{OH}))$ e gibbsita $\left(\mathrm{Al}(\mathrm{OH})_{3}\right)$ - podem adsorver íons de $\mathrm{Cr}(\mathrm{VI})$ e ter sua eficiência aumentada em ambientes ácidos (ZACHARA et al. 1988, RAI et al. 1989, FENDORF 1995, AJOUYED et al. 2010, JIANG et al. 2014). Para as espécies de Cr(III), em particular, o fenômeno de adsorção é favorecido com o aumento do $\mathrm{pH}$ e menor competição dada pela presença de cátions inorgânicos ou orgânicos (EARY \& RAI 1989, STANIN \& PIRNIE 2004).

$\mathrm{Na}$ adsorção, os íons metálicos na forma de cátions ligam-se mais facilmente aos componentes do solo, que são naturalmente carregados negativamente, como alguns argilominerais e substâncias húmicas (EARY \& RAI 1989, STANIN \& PIRNIE 2004). Portanto, materiais ricos em substâncias húmicas, como a turfa, também podem ser usados em tratamento de solos e águas contaminadas com metais tóxicos (ZHILIN et al. 2004, RIZZUTI et al. 2017, SANTOS et al. 2018).

Cabe ressaltar que tanto a sorção como a complexação orgânica (que será descrita a seguir), são reações que imobilizam o $\mathrm{Cr}(\mathrm{VI})$, porém não alteram sua toxidade. No entanto podem diminuir o risco deste íon se espalhar por grandes áreas. Dentre os constituintes do solo que podem adsorver o $\mathrm{Cr}(\mathrm{VI})$ destacam-se, por exemplo, os hidróxidos de $\mathrm{Al}$ e $\mathrm{Fe}$, que apresentam certa afinidade química com este íon por estarem positivamente carregados em condições de pH de 2 a 7 (FENDORF 1995).

\subsection{Complexação}

Reações de complexação ocorrem entre um cátion metálico (M) e um ligante iônico ou mole- 
cular (L) pela doação ao metal, por ligação covalente, de um ou mais pares de elétrons deste ligante (ATKINS \& JONES 2012). Quando o ligante doa mais do que um par de elétrons ao cátion metálico é chamando polidentado e, se este ligante se reorganiza como uma estrutura anelar envolvendo o íon metálico, é chamado de agente quelante (SKOOG et al. 2004). Esses complexos formados por metais ligados a agentes quelantes são chamados quelatos e apresentam elevada estabilidade.

O íon metálico pode formar complexos organometálicos ao reagir com a matéria orgânica do solo e, dentre os possíveis ligantes orgânicos, destacam-se a matéria orgânica suspensa (fração retida em filtro com $0,45 \mu \mathrm{m}$ ) e/ou sedimentável, além do carbono orgânico dissolvido (que passa em filtro de $0,45 \mu \mathrm{m}$ ) (SPARKS 2003). As substâncias húmicas ou ainda, o carbono orgânico dissolvido, possuem grupos funcionais que atuam como agentes redutores do $\mathrm{Cr}(\mathrm{VI})$, ou como agentes quelantes em reações de complexação com o Cr(III) (SPARKS 2003, TADINI et al. 2014). Os ácidos húmicos encontrados nas substâncias húmicas, por exemplo, podem reduzir o $\mathrm{Cr}(\mathrm{VI})$ à sua forma menos tóxica trivalente. Em seguida, o $\mathrm{Cr}$ (III) pode ser complexado pelos grupos carboxílicos dos ácidos húmicos, limitando sua disponibilidade no meio pela formação do complexo organometálico (SPARKS 2003).

$\mathrm{O}$ Cr(III) pode se ligar às substâncias húmicas por meio de complexos de esfera interna e externa (OTHA et al. 2012). Os complexos de esfera externa envolvem interações eletrostáticas rápidas, reversíveis, e ocorrem apenas em superfícies de cargas opostas, sendo relativamente fracos em comparação aos complexos de esfera interna, que fazem ligações covalente ou iônica (SPARKS 2003). A complexação da esfera interna é geralmente mais lenta do que a complexação da esfera externa e, muitas vezes, não é reversível. Este tipo de complexação pode aumentar, reduzir, neutralizar ou reverter a carga do íon, independentemente da carga original (SPARKS 2003).

Preferencialmente, os ácidos húmicos atuam como agentes quelantes para a espécie trivalente do cromo, formando complexos de esfera externa com o metal hidratado ou de esfera interna a partir de grupos carboxílicos (FUKUSHIMA et al. 1995). Por ser comumente encontrado sob forma de óxidos e hidróxidos insolúveis, o $\mathrm{Cr}$ (III) é pouco observado em águas subterrâneas (FRANCO 2008). Entretanto, com a formação do complexo orgânico sua mobilidade pode aumentar, assim como sua concentração iônica (OTHA et al. 2012).

\subsection{Precipitação e dissolução}

As reações de precipitação e dissolução afetam a solubilidade e a mobilidade do cromo em solo e água (KIMBROUGH et al. 1999, STANIN \& PIRNIE 2004). A maioria das espécies solúveis de Cr(III) é instável e não é comum ocorrer no ambiente, diferente dos ânions de $\mathrm{Cr}(\mathrm{VI})$, caracterizados pela elevada mobilidade (KIMBROUGH et al. 1999). Quando disponíveis no meio aquoso, os cátions metálicos podem formar fortes ligações com o oxigênio e dividir ou decompor uma molécula de água, formando uma nova espécie de metal iônico (um hidróxido ou óxido) e prótons. Este processo, chamado hidrólise, afeta não só a forma química do metal, como também as características de adsorção e troca iônica, podendo impactar sua reatividade com outras espécies solúveis (ESSINGTON 2005). Assim sendo, o cromo hexavalente hidrolisa-se facilmente em meio aquoso formando oxiânions estáveis (ESSINGTON 2005). Em concentrações menores que $0,01 \mathrm{~mol} \mathrm{~L}^{-1}$, verifica-se a existência de formas iônicas como $\mathrm{CrO}_{4}{ }^{2-}$ (cromato), $\mathrm{HCrO}_{4}^{-}$ (hidrocromato) e $\mathrm{Cr}_{2} \mathrm{O}_{7}^{2-}$ (dicromato), sendo o equilíbrio entre estes elementos dependente do $\mathrm{pH}$ (eq. 1) (PARK \& JANG 2002, WANG et al. 2016).

$2 \mathrm{CrO}_{4}^{2-}($ aq. $)+2 \mathrm{H}^{+}($aq. $) \rightleftharpoons 2 \mathrm{HCrO}_{4}^{-}(a q.) \rightleftharpoons \mathrm{Cr}_{2} \mathrm{O}_{7}^{2}($ aq. $)+\mathrm{H}_{2} \mathrm{O}(\mathrm{l})$

$\mathrm{O} \mathrm{Cr}(\mathrm{VI})$ na forma de $\mathrm{CrO}_{4}{ }^{2-}$ existe em soluções alcalinas ou neutras e, inicialmente, transforma-se em $\mathrm{HCrO}_{4}{ }^{2-}$ em valores baixos de $\mathrm{pH}$ (FRANCO 2008). Ainda em meio ácido, a espécie $\mathrm{HCrO}_{4}{ }^{2-}$ é transformada em $\mathrm{Cr}_{2} \mathrm{O}_{7}{ }^{2-}$, sendo esta última predominante em $\mathrm{pH}$ menor que 2 (WANG et al. 2016). Em valores de pH entre 6 e 8 e concentrações de $1 \mathrm{mg} \mathrm{L}^{-1}$, o íon $\mathrm{CrO}_{4}^{-}$é predominante e fornece uma coloração amarelada à solução aquosa; já em concentrações maiores, as espécies $\mathrm{HCrO}_{4}{ }^{2-} \mathrm{e}$ $\mathrm{Cr}_{2} \mathrm{O}_{7}{ }^{2-}$ são dominantes, e ambos transmitem uma forte coloração alaranjada ao líquido (PALMER \& PULS 1994, KIMBROUGH et al. 1999).

Apesar da elevada mobilidade de $\mathrm{Cr}(\mathrm{VI})$, BARON et al. (1996) identificaram dois precipitados distintos de $\mathrm{Fe}(\mathrm{III})$ com cromato: $\mathrm{KFe}_{3}\left(\mathrm{CrO}_{4}\right)_{2}(\mathrm{OH})_{6}$ e $\mathrm{KFe}\left(\mathrm{CrO}_{4}\right)_{2} \cdot 2 \mathrm{H}_{2} \mathrm{O}$. Os autores utilizaram técnicas de microscopia eletrônica e de difração de raios X no estudo de solos contaminados com soluções da indústria de cromação. Eles concluíram que a formação desses precipitados ocorreu em condições ácidas, uma vez que o $\mathrm{pH}$ da água adjacente era de 2,3. OLAZABAL et al. (1997) também constataram a formação de precipi- 
tados em estudos sobre o equilíbrio de sistemas de $\mathrm{Cr}(\mathrm{VI}) / \mathrm{Fe}(\mathrm{III}) / \mathrm{H}_{2} \mathrm{O}$, utilizando a espectroscopia de fotoelétrons de raios $\mathrm{X}$ para definir a estequiometria e as constantes de solubilidade dos precipitados formados em $\mathrm{pH}$ de 1 a 9. Os autores verificaram que sob condições ambientais e $\mathrm{pH}$ distintos, três precipitados se formam: $\mathrm{FeOHCrO}_{4} \mathrm{em} \mathrm{pH}$ entre 1,5 e 2,5; $\mathrm{FeOHCrO}_{4} \cdot 2 \mathrm{Fe}(\mathrm{OH})_{3}$ em $\mathrm{pH}$ entre 2,53,5 e $\mathrm{Fe}(\mathrm{OH})_{3}$ em $\mathrm{pH}$ maior que 3,5. Eles também notaram que a presença do íon cromato em meios com $\mathrm{pH}$ entre 1,5 e 3,5 afeta a solubilidade do ferro, induzindo à formação de precipitados de cromato/ ferro.

Quanto ao $\mathrm{Cr}(\mathrm{III})$, verifica-se que as formas de $\mathrm{Cr}^{3+}(\mathrm{aq})$ e $\mathrm{CrOH}^{2+}(\mathrm{aq})$ são comumente encontradas em meio ácido, enquanto as espécies de $\mathrm{Cr}(\mathrm{OH})_{3}{ }_{3}(\mathrm{~s})$ e $\mathrm{Cr}(\mathrm{OH})_{4}{ }^{-}(\mathrm{aq})$ em meio alcalino; já o $\mathrm{Cr}(\mathrm{OH})_{2}{ }^{+}(\mathrm{aq})$ ocorre com maior frequência em intervalo de $\mathrm{pH}$ de 6 a 8 (ESSINGTON 2005). Em altas concentrações, os íons de $\mathrm{Cr}$ (III) fornecem uma coloração esverdeada para o meio líquido (CALDER 1988, PALMER \& WITTBRODT 1991).

Os produtos da hidrólise de $\mathrm{Cr}(\mathrm{III})$ possibilitam a formação de sólidos que, consequentemente, diminuem a concentração do metal em solução. $\mathrm{O} \mathrm{Cr}(\mathrm{III})$ pode ser precipitado sob as formas de $\mathrm{Cr}(\mathrm{OH})_{3}{ }^{0}(\mathrm{~s}), \mathrm{FeCr}_{2} \mathrm{O}_{4}(\mathrm{~s})$ ou $\mathrm{Fe}_{\mathrm{x}} \mathrm{Cr}_{\mathrm{y}}(\mathrm{OH})_{3}(\mathrm{~s})$, cujo mecanismo pode sofrer a influência do $\mathrm{pH}$, presença de outros íons e da complexação com a matéria orgânica (RICHARD \& BOURG 1991). Com o aumento do $\mathrm{pH}$, aumenta-se a concentração de ânions $\mathrm{OH}^{-}$e, consequentemente, as reações de precipitação com Cr(III) (HAWLEY et al. 2004). Na presença de sais de ferro, a formação do hidróxido misto entre $\mathrm{Cr}(\mathrm{III})$ e ferro $\left(\mathrm{Fe}_{\mathrm{x}} \mathrm{Cr}_{\mathrm{y}}(\mathrm{OH})_{3}\right)$ torna-se um dos meios para controlar a fração solúvel de cromo em ambientes naturais, visto que este composto possui uma solubilidade inferior, se comparado ao hidróxido de cromo insolúvel e amorfo $\left(\mathrm{Cr}(\mathrm{OH})_{3}{ }^{0}\right)$ (RAI et al. 1989). Já na presença de carbono orgânico dissolvido, como os ácidos húmicos e fúlvicos, pode ocorrer a complexação com o $\mathrm{Cr}$ (III) dissolvido e, nesse caso, dificultar a remoção do metal por precipitação (HAWLEY et al. 2004, GUSTAFSSON et al. 2014).

A possibilidade de formar precipitados sob condições ambientais é de suma importância para o estudo de remediação de áreas contaminadas, uma vez que contribui com a diminuição do transporte de metais e, consequentemente, com a sua dispersão.

\subsection{Reações de oxirredução}

O estado de oxidação do cromo pode ser alterado dependendo das condições redutoras ou oxidantes em que está inserido. O potencial redox (doação ou aceitação de elétrons entre espécies) é influenciado por duas variáveis principais: o Eh (condições redutoras ou oxidantes) e pH (condições ácidas ou básicas) (RAI et al. 1989, ESSINGTON 2005). Pela análise do diagrama de Pourbaix do cromo (Figura 1) verifica-se que em condições ácidas $(\mathrm{pH}<6)$ e redutoras (baixo Eh), o $\mathrm{Cr}(\mathrm{III})$ é a espécie mais estável e, em sistemas alcalinos, ele ocorre na forma de hidróxidos $\left(\mathrm{Cr}(\mathrm{OH})_{3}\right)$ (ESSINGTON 2005).

As espécies reduzidas prevalecem em condições subóxicas e anóxicas, sob a forma de cátions $\mathrm{Cr}^{3+}, \mathrm{Cr}(\mathrm{OH})^{2+}, \mathrm{Cr}(\mathrm{OH})_{2}{ }^{+}$e ânion $\mathrm{Cr}(\mathrm{OH})_{4}^{-}$, o último em altos valores de $\mathrm{pH}$ (PALMER \& WITTBRODT 1991). Assim sendo, o Cr(III) é anfotérico e pode existir como cátions e ânions em função das condições do pH (ácidas ou básicas) (ESSINGTON 2005). Em condições oxidantes (Eh elevado) os ânions cromato $\left(\mathrm{HCrO}_{4}^{-}\right.$e $\left.\mathrm{CrO}_{4}{ }^{2-}\right)$ e dicromato $\left(\mathrm{HCr}_{2} \mathrm{O}_{7}^{-} \mathrm{e} \mathrm{Cr}_{2} \mathrm{O}_{7}^{2-}\right)$ são dominantes (RAI et al. 1989, ESSINGTON 2005, CAO \& ZHANG 2006). Em pH igual a 4,5 as espécies $\mathrm{CrO}_{4}{ }^{2-}$ e $\mathrm{Cr}_{2} \mathrm{O}_{7}{ }^{2-}$ se cruzam na linha limítrofe, isso quer dizer que em $\mathrm{pH}<4,5$ serão predominantes os compostos de cromato e, $\mathrm{pH}>4,5$ os ânions oxidantes de dicromato (KIMBROUGH et al. 1999, ESSINGTON 2005).

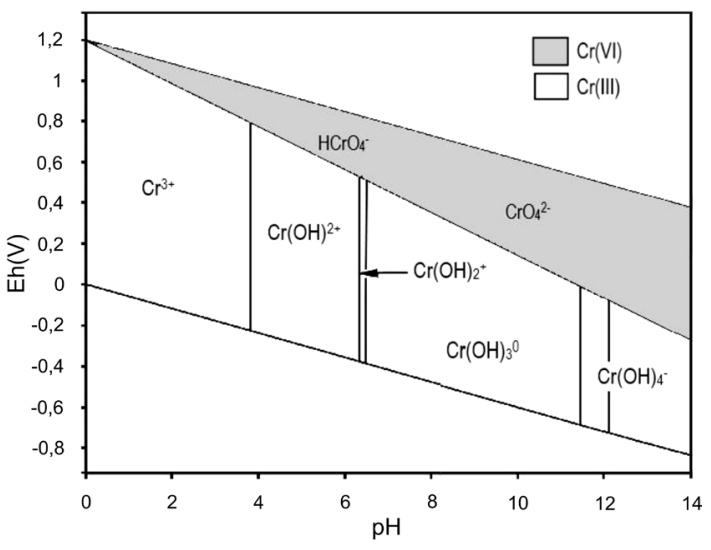

FIGURA 1 - Diagrama de Eh-pH do cromo em solução aquosa a $25^{\circ} \mathrm{C}$ e 1 atm. A área superior de cor cinza corresponde aos compostos de $\mathrm{Cr}(\mathrm{VI})$ e a área central de cor branca corresponde aos compostos de Cr(III). (Adaptado de PALMER \& WITTBRODT 1991). 
O Cr(VI), por se tratar de um forte agente oxidante, pode ser reduzido à forma trivalente (Cr(III)) na presença de doadores de elétrons (PALMER \& PULS 1994). Este processo depende do $\mathrm{pH}$, concentração de oxigênio, presença de redutores e outros elementos que atuam como ligantes ou catalisadores (STANIN \& PIRNIE 2004). Nas condições subóxicas e anóxicas, os processos de redução das formas de cromato são controladas por agentes como íons de $\mathrm{Fe}(\mathrm{II})$, minerais ferrosos, sulfetos e matéria orgânica, tanto em solo quanto em água (PALMER \& PULS 1994, STANIN \& PIRNIE 2004).

Em condições oxidantes, o cromo trivalente, por sua vez, pode ser oxidado à forma hexavalente na presença de óxidos e hidróxidos de manganês(III) e (IV) (RAI et al. 1989, PALMER \& WITTBRODT 1991). Os óxidos de Mn(III) e (IV), e o oxigênio molecular são os únicos agentes capazes de oxidar o $\mathrm{Cr}(\mathrm{III})$ a (VI) em sistemas naturais (EARY \& RAI 1987, RAI et al. 1989, FENDORF 1995, KIM et al. 2002). O oxigênio dissolvido pode vir a oxidar pequenas quantidades de $\mathrm{Cr}(\mathrm{III})$ se o meio apresentar $\mathrm{pH}$ maior que 9 (FENDORF \& ZAZOSKI 1992). Entretanto, esta reação possui velocidade lenta, se comparada a reações mais rápidas que envolvem os processos de adsorção, precipitação e a presença de óxidos de manganês sendo este último um oxidante mais importante em águas subterrâneas (RAI et al. 1989, RICHARD \& BOURG 1991). A reação de oxidação de $\mathrm{Cr}(\mathrm{III})$ por óxidos de manganês(IV) está ilustrada na equação 2 (APTE et al. 2006).

$\mathrm{Cr}^{3+}($ aq. $)+1,5 \mathrm{MnO}_{2}(\mathrm{~s})+\mathrm{H}_{2} \mathrm{O}(\mathrm{l}) \rightleftharpoons \mathrm{HCrO}_{4}($ aq. $)+1,5 \mathrm{Mn}^{2+}$ (aq. $)+\mathrm{H}^{+}$(aq. $)$

Essa reação pode sofrer influência de outras variáveis como a superfície de adsorção de $\mathrm{MnO}_{2}$ e o pH do meio (FENDORF \& ZAZOSKI 1992, FENDORF 1995, APTE et al. 2006). AMACHER \& BAKER (1982) verificaram, por exemplo, que a taxa de oxidação de $\mathrm{Cr}$ (III) por birnessita $\left(\delta-\mathrm{MnO}_{2}\right)$ em solução aumentou até atingir $\mathrm{pH} 5,5$, e não sofreu alterações com valores maiores de $\mathrm{pH}$. Entretanto, os autores notaram que na reação com a pirolusita $\left(\beta-\mathrm{MnO}_{2}\right) \mathrm{em}$ solo, essa taxa diminuiu com o aumento do $\mathrm{pH}$.

FENDORF (1995) verificou que a presença de um precipitado de hidróxido de cromo $\left(\mathrm{Cr}(\mathrm{OH})_{3}\right.$. $\mathrm{nH}_{2} \mathrm{O}$ ) na superfície do óxido de manganês inibiu a oxidação de $\mathrm{Cr}(\mathrm{III})$ em solução com $\mathrm{pH}$ 4,0. O precipitado pode formar um complexo de esfera interna com o óxido de manganês e restringir a oxi- dação de duas formas: como um sumidouro estável para o $\mathrm{Cr}$ (III) oxidável e solúvel e, formando uma barreira física entre o $\mathrm{Cr}$ (III) aquoso e a superfície do óxido de manganês (FENDORF et al. 1992). Todavia, a oxidação dependerá da taxa de dissolução de $\mathrm{Cr}(\mathrm{III})$ : se o metal estiver na presença de agentes quelantes orgânicos, sua dissolução pode aumentar, contribuindo para oxidação (FENDORF 1995).

Em estudo realizado com $\mathrm{Cr}(\mathrm{OH})_{3}$ e $\beta-\mathrm{MnO}_{2}$, APTE et al. (2006) notaram que o processo de oxidação envolve a adsorção dos íons Cr(III) dissolvidos na superfície do mineral, seguido da liberação da forma hexavalente de cromo juntamente com íons manganês(II). Os autores verificaram que as espécies formadas de $\mathrm{Cr}(\mathrm{VI})$ encontravam-se adsorvidas na superfície de $\beta-\mathrm{MnO}_{2}$, conforme o $\mathrm{pH}$ era reduzido de 8,0 a 2,0. Dessa forma, os autores verificaram uma queda na produção de íons de $\mathrm{Cr}(\mathrm{VI}) \mathrm{e}$, com o aumento do $\mathrm{pH}$, a adsorção de $\mathrm{Cr}(\mathrm{III})$ na superfície de $\beta-\mathrm{MnO}_{2}$.

\section{INTERAÇÃO DOS CONSTITUINTES DO SOLO NA REMOÇÃO DE CROMO(VI)}

Áreas contaminadas com $\mathrm{Cr}(\mathrm{VI})$ são tratadas com tecnologias que visam reduzir a toxicidade, remover e/ou conter o contaminante, evitando assim os possíveis riscos que o seu espalhamento pode causar, incluindo sua reoxidação à forma hexavalente, após redução para a espécie trivalente (MULLIGAN et al. 2001, HAWLEY et al. 2004). Segundo XIAO et al. (2012), a redução de $\mathrm{Cr}(\mathrm{VI})$ em solos naturais é resultado dos efeitos combinados de matéria orgânica dissolvida, teor de $\mathrm{Fe}(\mathrm{II})$, $\mathrm{pH}$, comunidade bacteriana e distribuição de tamanho das partículas do solo.

As estratégias de remediação das matrizes contaminadas incluem basicamente os processos que influenciam na especiação ou forma do cromo no ambiente, já citados: adsorção; precipitação, complexação e oxirredução (HAWLEY et al. 2004). Em solos, a redução do metal seguido de precipitação pode ocorrer naturalmente, resultado das próprias condições do meio, sem necessitar de agentes redutores adicionais (RAI et al. 1989, RICHARD \& BOURG 1991, FRANCO 2008, HORI et al. 2015). ZHANG et al. (2019) também verificaram que a adsorção do $\mathrm{Cr}(\mathrm{VI})$ em um solo (black soil) precede a sua redução a $\mathrm{Cr}$ (III) sendo, portanto, o modelo cinético em duas etapas a mais adequada para representar esses mecanismos. 
Para águas subterrâneas, um dos métodos mais viáveis para remoção do elemento também é a inserção de agentes redutores no meio contaminado (HWANG et al. 2002, GRÖHLICH et al. 2017). Este processo promove a redução de $\mathrm{Cr}(\mathrm{VI})$ a $\mathrm{Cr}(\mathrm{III})$, permitindo a atenuação da mobilidade do metal e transformação para a espécie menos tóxica (HWANG et al. 2002, OWLAND et al. 2008).

Portanto, para se aplicar um processo de tratamento, deve-se atentar a três principais condições: (1) evitar a formação de subprodutos indesejáveis no processo de redução (como formas oxidadas de certos agentes redutores: nitratos, óxidos de Fe(III), compostos contendo enxofre); (2) mudanças bruscas nas condições redox e acidez, que podem inibir a precipitação de $\mathrm{Cr}$ (III) e; (3) imobilização do $\mathrm{Cr}$ (III) para evitar que seja oxidado novamente a $\mathrm{Cr}(\mathrm{VI})$ por óxidos de manganês (JAMES et al. 1997).

\subsection{Matéria orgânica x Cromo(VI)}

A matéria orgânica encontrada no solo inclui todos os compostos orgânicos ali presentes, desde restos de plantas a substâncias orgânicas totalmente transformadas, além de produtos microbianos (KÖGEL-KNABNER 2017). Para BOHN et al. (2001) a matéria orgânica do solo corresponde à fração contendo carbono, exceto carbonatos, incluindo, portanto, os compostos humificados e não humificados. O termo "húmus" representa a fração mais estável de decomposição da matéria orgânica. De acordo com SPARKS (2003), o húmus inclui substâncias húmicas $(\mathrm{SH})$ mais produtos de ressíntese de microrganismos que são estáveis e fazem parte do solo.

Os compostos não humificados incluem, por exemplo, ácidos orgânicos de massa molecular baixa; carboidratos simples e polissacarídeos; açúcares; aminoácidos; peptídeos e proteínas; lipídios e fosfolipídeos; ácidos nucleicos e lignina (ESSINGTON 2005). O húmus inclui as sustâncias húmicas $(\mathrm{SH})$ e outros produtos considerados como as frações mais estáveis, derivadas da síntese microbiana (BOHN et al. 2001, SPARKS 2003).

As substâncias húmicas são macroligantes naturais de alto peso molecular formados por reações de síntese secundária no solo (SPARKS 2003). O material quando solubilizado, apresenta cores que vão de marrom à preta e, possui três frações representadas por sua solubilidade em meio ácido e básico: os ácidos fúlvicos, húmicos e humina (WITTBRODT \& PALMER 1996, SPARKS 2003, ZHILIN et al. 2004). A fração de ácidos hú- micos é solúvel em $\mathrm{pH}>2$ e precipitada em valores abaixo deste. Já os ácidos fúlvicos são solúveis em qualquer valor de $\mathrm{pH}$, possuindo um baixo peso molecular se comparado à humina, caracterizada pelo alto peso molecular e insolubilidade em todo valor de pH (WITTBRODT \& PALMER 1996, KÖGEL-KNABNER \& RUMPEL 2018).

As substâncias húmicas são um dos principais constituintes dos solos de turfa, um material de baixo custo, altamente utilizado no tratamento de soluções contaminadas por metais (BROWN et al. 2000, RIZZUTTI et al. 2017, SANTOS et al. 2018). A turfa é um sedimento natural de origem vegetal advinda de áreas onde a produção de matéria orgânica excede a decomposição, acarretando na sua acumulação e formação do sedimento de turfa (MOORE 1989, PAGE et al. 2006, CERQUEIRA et al. 2012).

A turfa possui uma composição química complexa e influenciada pela região de sua formação. Se originada em áreas tropicais com alta pluviosidade e temperatura, sua decomposição advém de detritos de plantas lenhosas e, se formada em regiões temperadas, ela deriva da decomposição de musgos e outros briófitos, juncos, gramíneas, arbustos ou árvores (COCOZZA et al. 2003). Todavia, os solos de turfa possuem constituintes complexos, normalmente representados por matéria orgânica (betumes - ácidos graxos, ceras e esteroides), carboidratos (celulose e proteínas), ligninas e substâncias húmicas (BROWN et al. 2000, FRANCHI et al. 2006).

Estudos demonstram que a turfa é eficaz na remoção de $\mathrm{Cr}(\mathrm{VI})$ em soluções aquosas (ZHILIN et al. 2004, CERQUEIRA et al. 2012, HENRYK et al. 2016, RIZZUTTI et al. 2017, SANTOS et al. 2018) bem como as suas respectivas frações húmicas, destacando-se maior eficiência para os ácidos fúlvicos, se comparados aos húmicos e humina (KASIULIENE et al. 2018, ALDMOUR et al. 2019). Entretanto, torna-se complexo comparar as pesquisas quando as características deste material se diferenciam (tipo de turfa, tratamento ácido antes do contato com o metal, metodologias distintas). Cada tratamento dado ao material pode alterar o processo de remoção dos metais; no entanto, os principais mecanismos de remoção se dão pelos processos de redução e adsorção/complexação com a fração orgânica.

Em estudo realizado por ECKERT et al. (1990), a porcentagem de remoção de $\mathrm{Cr}(\mathrm{VI})$ (em $72 \mathrm{~h}$ ) por ácidos fúlvicos foi mais eficiente $\mathrm{em} \mathrm{pH}$ 2,0 (94\%) do que em pH 4,0 (88\%). Resultados 
semelhantes também foram observados por CERQUEIRA et al. (2012), que verificaram maior eficiência (em $50 \mathrm{~h}$ ) para a turfa in natura em $\mathrm{pH}$ $2,0(70 \%)$ do que em pH 6,0 (13\%). Em quatro tipos de turfas analisadas por RIZZUTTI et al. (2017), a remoção de $\mathrm{Cr}(\mathrm{VI})$ em soluções aquosas ácidas $(\mathrm{pH} 2,0)$ foi mais eficiente com a utilização de turfas com menor quantidade de ácidos húmicos em relação aos ácidos fúlvicos.

SANTOS et al. (2018) realizaram um estudo de remoção de $\mathrm{Cr}(\mathrm{VI})$ em água subterrânea usando turfa tropical. Os autores verificaram uma remoção de $1,1 \mathrm{mg} \mathrm{g}^{-1}$ de $\mathrm{Cr}(\mathrm{VI})$ que correspondia à uma eficiência de $95 \%$ em pH 2,0 alcançada em 24 h, e de $2,0 \mathrm{mg} \mathrm{g}^{-1}$ ( $41 \%$ de eficiência) em pH 5,0 por um período de 5 dias. De acordo com MACHADO et al. (2011), o $\mathrm{Cr}(\mathrm{VI})$ pode ser reduzido à forma trivalente por meio da oxidação do carbono orgânico da turfa (eq. 3) e, em seguida, sofrer precipitação, ou ainda ser adsorvido/complexado pelos componentes inorgânicos e orgânicos do material.

$3 \mathrm{C}^{0}(\mathrm{~s})+2 \mathrm{Cr}_{2} \mathrm{O}_{7}^{2-}(\mathrm{aq})+16 \mathrm{H}^{+}(\mathrm{aq}) \rightleftharpoons 4 \mathrm{Cr}^{3+}(\mathrm{aq})+3 \mathrm{CO}_{2}(\mathrm{~g})+8 \mathrm{H}_{2} \mathrm{O}(\mathrm{l})$

As substâncias húmicas também são responsáveis pelo processo de redução do $\mathrm{Cr}(\mathrm{VI})$ a $\mathrm{Cr}(\mathrm{III})$, cujo mecanismo pode atuar em conjunto ou separadamente com a adsorção de $\mathrm{Cr}(\mathrm{VI}) / \mathrm{Cr}$ (III) ou complexação de $\mathrm{Cr}$ (III). Vários estudos sobre a redução do metal à forma trivalente (ZHILLIN et al. 2004, AGRAWAL et al. 2009, CERQUEIRA et al. 2012, JIANG et al. 2014, SANTOS et al. 2018) demonstraram que a reação (eq. 3) acontece de forma lenta e possui relação forte e inversa com os valores de $\mathrm{pH}$ do meio. Contudo, a taxa de redução de $\mathrm{Cr}(\mathrm{VI})$ pode ser modificada na presença de $\mathrm{Fe}(\mathrm{II})$ e, neste caso, o $\mathrm{Cr}(\mathrm{VI})$ será reduzido mais rapidamente (WITTBRODT \& PALMER 1996, ZHILLIN et al. 2004).

\subsection{Ferro(II) x Cromo(VI)}

Em águas, sedimentos ou solos contaminados, os íons de $\mathrm{Fe}$ (II) são considerados um dos principais agentes redutores de $\mathrm{Cr}(\mathrm{VI})$ (BUERGE \& HUG 1997, SCHLAUTMAN \& HAN 2001, BROOKSHAW et al. 2014). Uma vez no ambiente, os íons de Fe(II) participam de uma reação de oxirredução relativamente rápida com o Cr(VI) (EARY \& RAI 1988, KWAK et al. 2018). O processo é influenciado pela temperatura do meio e por valores de $\mathrm{pH}$ que variam de moderados a ácidos (EARY \& RAI 1988, 1989;
BUERGE \& HUG 1997; VINUTH et al. 2015; HU et al. 2019).

Os produtos formados pela oxirredução do ferro mudam, dependendo do $\mathrm{pH}$ (BUERGE \& HUG 1997): em condições ácidas, como $\mathrm{pH}$ 2,0, o agente oxidante passa a consumir os prótons do meio para realizar a redução de $\mathrm{Cr}(\mathrm{VI})$ (eq. 4). Com o pH entre 4,0 e 6,0, estima-se a formação de hidróxidos de $\mathrm{Fe}(\mathrm{III})$ (eq. 5) e, conforme o $\mathrm{pH}$ aumenta, há consumo de $\mathrm{OH}^{-}$pela precipitação de $\mathrm{Fe}(\mathrm{III}) / \mathrm{Cr}(\mathrm{III})$ e aumento de prótons na solução (eq. 6).

$3 \mathrm{Fe}^{2+}(a q)+\mathrm{HCrO}_{4}^{-}(a q)+7 \mathrm{H}^{+}(a q) \rightleftharpoons 3 \mathrm{Fe}^{3+}(a q)+\mathrm{Cr}^{3+}(a q)+4 \mathrm{H}_{2} \mathrm{O}(\mathrm{l})$

$3 \mathrm{Fe}^{2+}(a q)+\mathrm{HCrO}_{4}^{-}(\mathrm{aq})+3 \mathrm{H}_{2} \mathrm{O}(\mathrm{l}) \rightleftharpoons 3 \mathrm{Fe}(\mathrm{OH})_{2}^{+}(a q)+\mathrm{CrOH}^{2+}(a q)$

$3 \mathrm{Fe}^{2+}(\mathrm{aq})+\mathrm{HCrO}_{4}^{-}(\mathrm{aq})+8 \mathrm{H}_{2} \mathrm{O}(\mathrm{l}) \rightleftharpoons \mathrm{Fe}_{3} \mathrm{Cr}(\mathrm{OH})_{12}(\mathrm{~s})+5 \mathrm{H}^{+}(\mathrm{aq})$

Estudos utilizando concentrações $<1 \mathrm{mg} \mathrm{L}^{-1}$ de $\mathrm{Fe}(\mathrm{II})$ e $\geq 1 \mathrm{mg} \mathrm{L}^{-1} \mathrm{Cr}(\mathrm{VI})$ apresentam completa redução do metal hexavalente em baixos valores de pH (30 min de contato) e, reação incompleta ou lenta a partir de pH 4,0 (EARY \& RAI 1988, BUERGE \& HUG 1997, MITRAKAS et al. 2011, VINUTH et al. 2015, HU et al. 2017). O aumento do $\mathrm{pH}$, por sua vez, tende a causar a rápida oxidação de $\mathrm{Fe}(\mathrm{II})$ a $\mathrm{Fe}(\mathrm{III})$ pelo oxigênio dissolvido, diminuindo a capacidade do agente redutor (BUERGE \& HUG 1997).

Não só os íons de Fe(II) em solução, mas os próprios minerais que contém $\mathrm{Fe}$ (II) podem participar do processo de redução de Cr(VI) (EARY \& RAI 1989, KIM et al. 2007, BROOKSHAW et al. 2014, KWAK et al. 2018). KWAK et al. (2018) e HU et al. (2019) avaliaram a redução de $\mathrm{Cr}(\mathrm{VI})$ pela montmorillonita $\left(\mathrm{M}_{\mathrm{x}}\left(\mathrm{Si}_{8}\right)\right.$ $\left.\mathrm{Al}_{3,2} \mathrm{Fe}_{0,2} \mathrm{Mg}_{0,6} \mathrm{O}_{20}(\mathrm{OH})_{4}\right)$ e ferridrita $\left(\mathrm{Fe}(\mathrm{OH})_{3}\right.$. $\mathrm{nH}_{2} 0$ ), respectivamente. Nesse caso, os principais mecanismos de remoção foram por redução de $\mathrm{Cr}(\mathrm{VI})$ pelos íons remanescentes de $\mathrm{Fe}(\mathrm{II})$ na fase aquosa, por adsorção de $\mathrm{Cr}(\mathrm{VI})$ na superfície dos minerais e pela própria redução de $\mathrm{Cr}(\mathrm{VI})$ pelos íons de $\mathrm{Fe}(\mathrm{II})$ adsorvidos ao mineral.

Em solos ricos em minerais de $\mathrm{Fe}(\mathrm{II}) \mathrm{e}$ $\mathrm{Fe}(\mathrm{III})$, a adsorção de $\mathrm{Cr}(\mathrm{VI})$ é um dos principais mecanismos para remoção do metal (AJOUYED et al. 2010). Reter as espécies de Cr(VI) por adsorção não significa reduzi-las à sua forma menos tóxica (Cr(III)) (HU et al. 2019), como é o caso de minerais ou precipitados de Fe(III) em solos tropicais. Contudo, a presença de matéria orgâni- 
ca possibilita a redução de $\mathrm{Fe}(\mathrm{III})$ a (II) e, neste caso, o $\mathrm{Cr}(\mathrm{VI})$ pode ser reduzido à sua forma trivalente (WITTBRODT \& PALMER 1996, HORI et al. 2015).

\subsection{Ferro(III) x Matéria Orgânica x Cromo(VI)}

Como foi visto, a presença de íons de $\mathrm{Fe}(\mathrm{II})$ em solos e águas proporciona um aumento na eficiência de remoção de $\mathrm{Cr}(\mathrm{VI})$. Não obstante, a presença de minerais e compostos de $\mathrm{Fe}(\mathrm{III})$ em solos também pode auxiliar na remoção do metal. Nesse caso, as substâncias orgânicas participam de uma reação de redução dos íons de $\mathrm{Fe}(\mathrm{III})$ a Fe(II) (HORI et al. 2015, CHEN et al. 2019) (eq.7).

$\mathrm{CH}_{3} \mathrm{COOH}(\mathrm{s})+8 \mathrm{Fe}^{3+}(a q)+2 \mathrm{H}_{2} \mathrm{O}(\mathrm{l}) \rightleftharpoons 2 \mathrm{CO}_{2}(\mathrm{~g})+8 \mathrm{Fe}^{2+}(\mathrm{aq})+8 \mathrm{H}^{+}(\mathrm{aq})$

O processo de redução de $\mathrm{Fe}(\mathrm{III})$ a (II) por compostos orgânicos é influenciado pelo $\mathrm{pH}$ do meio. Em valores maiores que 3,5 a redução torna-se menor pela formação de complexos Fe(III)matéria orgânica, como observado por DEIANA et al. (1995). Segundo os autores, a maior eficiência no processo de redução de $\mathrm{Fe}(\mathrm{III})$ por ácidos húmicos ocorre em pH 2,0 e 2,5 (70\%), quando comparado a valores de $\mathrm{pH}$ 3,0 e 3,5 (39\%). WITTBRODT \& PALMER (1996) também observaram que altos valores de $\mathrm{pH}$ inibem a redução de $\mathrm{Fe}$ (III) a (II). Nesse caso, os íons de Fe(III) em solução tornam-se limitados pela formação de $\mathrm{Fe}(\mathrm{OH})_{3}$ (amorfo) ou outros hidróxidos de Fe(III).

A associação com a matéria orgânica não acontece apenas com os íons de Fe(III). Diversos estudos indicam a ocorrência de um complexo $\mathrm{Fe}(\mathrm{II})$-orgânico estável (TÓMBACZ et al. 2004, JIANG et al. 2014, CHEN et al. 2015, BHATTACHARYYA et al. 2017, SODANO et al. 2017, YE et al. 2017). Além desse mecanismo, há a possibilidade da própria matéria orgânica dissolvida, como ácidos húmicos e fúlvicos, ser adsorvida por minerais ricos em $\mathrm{Fe}(\mathrm{II})$ e $\mathrm{Fe}(\mathrm{III})$, como hematita, magnetita e goethita (TÓMBACZ et al. 2004, YE et al. 2017, USMAN et al. 2018). É importante ressaltar que a complexação de $\mathrm{Fe}(\mathrm{II}) / \mathrm{Fe}$ (III) com as frações orgânicas é estável e a formação desses complexos diminui a concentração iônica destes metais em solução (JIANG et al. 2014).

Quando disponíveis em meio contaminado por cromo e em presença de matéria orgânica, os íons de $\mathrm{Fe}$ (III) participam de um ciclo catalítico em que as substâncias húmicas o reduzem a $\mathrm{Fe}(\mathrm{II})$ (eq. 7) que, em seguida, causa a redução de $\mathrm{Cr}(\mathrm{VI})$ a (III) (eq. 8) (WITTBRODT \& PALMER 1996). Essa reação ocorre nos primeiros 10 min e antes do ferro ser complexado (KWAK et al. 2018).

$6 \mathrm{Fe}^{2+}(a q)+\mathrm{Cr}_{2} \mathrm{O}_{7}^{2-}(\mathrm{aq})+14 \mathrm{H}^{+}(\mathrm{aq}) \rightleftharpoons 6 \mathrm{Fe}^{3+}(\mathrm{aq})+2 \mathrm{Cr}^{3+}(\mathrm{aq})+7 \mathrm{H}_{2} \mathrm{O}(\mathrm{l})$

A reação de oxirredução em um sistema com matéria orgânica-Fe(III)-Cr(VI) é dependente da concentração de $\mathrm{Fe}$ (III) (WITTBRODT \& PALMER 1996). ZHILIN et al. (2004) observaram um aumento significativo na taxa de redução de $\mathrm{Cr}(\mathrm{VI})$ após adição de sal de $\mathrm{Fe}(\mathrm{III})$ em solução contendo turfa. Os autores sugerem que o Fe(III), mesmo em pequenas concentrações, sofreu redução a (II) após oxidar as substâncias húmicas da turfa, permanecendo no ciclo de oxirredução entre substâncias húmicas-Fe(II)-Cr(VI). HORI et al. (2015) ao utilizarem sal de Fe(II) e ácidos húmicos também verificaram a redução $\mathrm{Cr}(\mathrm{VI})$ a (III); no entanto, os autores notaram que o $\mathrm{Fe}$ (III) gerado no processo foi novamente reduzido pelos ácidos húmicos em solução, produzindo novamente íons de $\mathrm{Fe}$ (II) com a mesma estrutura do sal adicionado no sistema.

Assim sendo, em solos contendo Fe(III) e $\mathrm{Fe}(\mathrm{II})$ pode-se considerar que o ciclo de oxirredução entre substâncias húmicas-Fe(II)-Cr(VI) também pode ocorrer. Tal fato também foi observado por HORI et al. (2015) usando um solo contendo ácidos húmicos, fúlvicos, $\mathrm{Fe}(\mathrm{II}), \mathrm{Fe}(\mathrm{III})$ e manganês. Os autores observaram que esse solo foi capaz de reduzir $86 \%$ de $\mathrm{Cr}(\mathrm{VI})$ em $124 \mathrm{~min}$ de contato, sem necessitar de agentes redutores adicionais. Nesse caso, a maior fração de ferro total no solo correspondia aos íons de Fe(III), enquanto o Fe(II) estava associado a minerais, necessitando de dissolução para participar da reação de redução de Cr(VI) (EARY \& RAI 1988, 1989). Entretanto, como os íons de $\mathrm{Fe}(\mathrm{III})$ possuem alta afinidade com os ácidos húmicos aniônicos do solo, estes foram reduzidos a $\mathrm{Fe}(\mathrm{II})$, permanecendo no ciclo de oxirredução de ácidos húmicos-Fe(II)-Cr(VI).

Além de auxiliar na redução de $\mathrm{Fe}(\mathrm{III})$ a $\mathrm{Fe}(\mathrm{II})$, as substâncias húmicas também participam da redução de $\mathrm{Cr}$ (VI) a (III). MAK \& LO (2011) verificaram que a redução do $\mathrm{Cr}(\mathrm{VI})$ ocorre tanto com ácidos húmicos quanto fúlvicos, mesmo na presença de íons de ferro. Entretanto, os autores notaram que na presença de ácidos húmicos e $\mathrm{Fe}(\mathrm{II})$, a remoção de $\mathrm{Cr}$ (VI) foi maior devido aos íons livres de $\mathrm{Fe}(\mathrm{II})$ em solução. Isto ocorreu porque provavelmente os ácidos fúlvicos eram adsorvidos nos óxidos de ferro, os quais possuem maior 
afinidade pelos ácidos e limitavam os íons de ferro em solução.

Apesar de várias pesquisas indicarem a possibilidade de ocorrer diversos mecanismos para reduzir o cromo hexavalente para a sua forma menos tóxica trivalente, estudos mais detalhados sobre a interação entre $\mathrm{Fe}(\mathrm{III})$ e frações húmicas na formação de $\mathrm{Fe}(\mathrm{II})$ em solos tropicais, como o Latossolo Vermelho, merecem mais atenção, uma vez que a maioria utiliza sais em seus testes.

O Latossolo Vermelho é um solo altamente intemperizado e encontrado em grande parte do território brasileiro, cuja textura é predominantemente argilosa (KER 1998). Esse solo também se caracteriza por apresentar elevadas concentrações de ferro (1 a $500 \mathrm{~g} \mathrm{~kg}^{-1}$ ), principalmente na forma de óxidos e oxi-hidróxidos, como hematita $\left(\alpha \mathrm{Fe}_{2} \mathrm{O}_{3}\right)$ e goethita $(\alpha-\mathrm{FeOOH})$, além de possuir outros minerais ricos em silício e alumínio, como caulinita $\left(\mathrm{Al}_{2} \mathrm{Si}_{2} \mathrm{O}_{5}(\mathrm{OH})_{4}\right)$ e gibbsita $\left(\mathrm{Al}(\mathrm{OH})_{3}\right)$ (INDA JR. 2002). Alguns destes óxidos e hidróxidos ocorrem sob forma de materiais amorfos e, até minerais altamente cristalizados (TREMOCOLDI 2003).

Os óxidos de ferro pedogênicos, como a goethita e a hematita, podem desempenhar um importante papel no comportamento físico e químico do solo, por apresentarem alta reatividade devido à sua elevada área superficial específica, bem como, pelo caráter variável de suas cargas superficiais (INDA JR \& KÄMPF 2005). Dentre estas propriedades, destaca-se a agregação do solo a partir da associação dos óxidos de ferro com outros minerais e compostos orgânicos, assim como a sorção com ânions e moléculas superficiais (INDA JR 2002).

Portanto, conhecer os principais mecanismos e reações químicas que envolvem os constituintes encontrados em solos tropicais e os contaminantes do meio, pode ser muito útil na escolha da técnica mais adequada para remediar áreas contaminadas com $\mathrm{Cr}(\mathrm{VI})$.

\section{CONCLUSÕES}

O comportamento e a toxicidade das duas espécies mais comuns de cromo no ambiente $(\mathrm{Cr}(\mathrm{VI})$ e Cr(III)) são muito distintas, o que torna essencial compreender quais agentes são capazes de interagir e interferir na sua mobilidade através do solo.

Em matrizes de solos, os processos de sorção e complexação que ocorrem, respectivamente, com as frações inorgânicas e orgânicas, são reações que imobilizam o cromo hexavalente mas não alteram sua toxicidade. Esses mecanismos também podem permitir que o íon sofra dissolução e atinja a água subterrânea, podendo ocasionar danos à saúde do homem, uma vez que diversas cidades utilizam a água subterrânea como principal fonte para abastecimento público e industrial.

Portanto, em estudos de áreas contaminadas com cromo é muito importante caracterizar o meio a fim de analisar a especiação desse metal nas condições encontradas no solo, como composição química, teor de matéria orgânica, $\mathrm{pH}$ etc. Dentre os principais agentes do solo capazes de favorecer a redução do $\mathrm{Cr}(\mathrm{VI})$ a $\mathrm{Cr}(\mathrm{III})$ estão a matéria orgânica e minerais com $\mathrm{Fe}$ (II).

Tanto o carbono da matéria orgânica quanto o $\mathrm{Fe}(\mathrm{II})$ são importantes agentes redutores de $\mathrm{Cr}(\mathrm{VI})$ a $\mathrm{Cr}$ (III). No entanto, íons de Fe(II) não são comumente encontrados em ambientes submetidos a forte intemperismo químico e sob condições oxidantes, como nos solos tropicais. Nesses solos, como no Latossolo Vermelho, predominam componentes ricos em $\mathrm{Fe}(\mathrm{III})$.

Apesar do $\mathrm{Fe}(\mathrm{III})$ não ser um agente capaz de reduzir o $\mathrm{Cr}(\mathrm{VI})$, a sua interação com a matéria orgânica (principalmente dissolvida) é capaz de formar facilmente $\mathrm{Fe}$ (II). Portanto, a presença desse íon e do carbono orgânico nos solos (que pode ser natural ou simplesmente adicionado na forma de corretivo como a turfa) leva à formação de íons de $\mathrm{Fe}(\mathrm{II})$ que, por sua vez, promovem a redução do $\mathrm{Cr}(\mathrm{VI})$.

Portanto, aproveitar os próprios componentes do solo como agentes facilitadores de reações de oxirredução do $\mathrm{Cr}(\mathrm{VI})$ para deixá-lo na forma menos tóxica e imobilizada, torna-se a estratégia de remediação mais sustentável - uma vez que se reduz o uso de reagentes químicos para promover a mesma ação. Dessa forma diminui-se também os riscos nocivos à saúde humana e ao ambiente, além de reduzir drasticamente o custo da operação de remediação.

\section{AGRADECIMENTOS}

Os autores agradecem a CAPES pela concessão de bolsa de Mestrado de Vanessa Maronezi e ao CNPq às bolsas de Iniciação Científica de Danielle Bittencourt Faria e Maria Isabel Garcia Rosa. Aos relatores da Revista do Instituto Geológico pelas valiosas sugestões apresentadas. 


\section{REFERÊNCIAS}

AGRAWAL, S.G.; FIMMEN, R.L.; CHIN, Y.P. 2009. Reduction of $\mathrm{Cr}(\mathrm{VI})$ to $\mathrm{Cr}(\mathrm{III})$ by $\mathrm{Fe}(\mathrm{II})$ in the presence of fulvic acids and in lacustrine pore water. Chemical Geology, 262(3-4): 328-335. https://doi. org/10.1016/j.chemgeo.2009.02.001

AJOUYED, O.; HUREL, C.; AMMARI, M.; ALLAL, L.B.; MARMIER, N. 2010. Sorption of $\mathrm{Cr}(\mathrm{VI})$ onto natural iron and aluminum (oxy)hydroxides: Effects of $\mathrm{pH}$, ionic strength and initial concentration. Journal of Hazardous Materials, 174(13): 616-622. https://doi.org/10.1016/j. jhazmat.2009.09.096

ALDMOUR, S.T.; BURKE, I.T.; BRAY, A.W.; BAKER, D.L.; ROSS, A.B.; GILL, F.L.; CIBIN, G.; RIES, M.E.; STEWART, D.I. 2019. Abiotic reduction of $\mathrm{Cr}(\mathrm{VI})$ by humic acids derived from peat and lignite: kinetics and removal mechanism. Environmental Science and Pollution Research, 26(5): $4717-$ 4729. https://doi.org/10.1007/s11356-0183902-1

AMACHER, M.C.; BAKER, D.E. 1982. Redox reactions involving chromium, plutonium, and manganese in soils. Institute for research on land and water research, Pennsylvania State University, University Park, PA, Final report, 1 July 1978 - 30 September 1981 https://doi.org/10.2172/5030864

APTE, A.; TARE, V.; BOSE, P. 2006. Extent of oxidation of $\mathrm{Cr}(\mathrm{III})$ to $\mathrm{Cr}(\mathrm{VI})$ under various conditions pertaining to natural environment. Journal of Hazardous Materials, 128(2-3): 164-174. https://doi. org/10.1016/j.jhazmat.2005.07.057

ATKINS, P.; JONES, L. 2012. Principios de química: questionando a vida moderna e o meio ambiente. Bookman, Porto Alegre, $5^{\mathrm{a}}$ ed., $874 \mathrm{p}$.

\section{ATSDR - AGENCY FOR TOXIC SUBSTANCES} AND DISEASE REGISTRY. 2012. Toxicological profile for chromium. U.S. Department of Health and Human Services, 502 p. https://www.atsdr.cdc. gov $/$ toxprofiles $/$ tp.asp?id $=62 \&$ tid $=17$
BARON, D.; PALMER, C.D.; STANLEY, J.T. 1996. Identification of two iron-chromate precipitates in a $\mathrm{Cr}(\mathrm{VI})$ contaminated soil. Environmental Science \& Technology, 30(30): 964-968. https://doi.org/10.1021/ es9504348

BHATTACHARYYA, A.; SCHMIDT, M.P.; STAVITSKI, E.; MARTÍNEZ, C.E. 2017. Iron speciation in peats: Chemical and spectroscopic evidence for the co-occurrence of ferric and ferrous iron in organic complexes and mineral precipitates. Organic Geochemistry, 115: 124-137. https://doi. org/10.1016/j.orggeochem.2017.10.012

BOHN, H.L.; MCNEAL, B.L.; O'CONNOR, G.A. 2001. Soil Chemistry. John Wiley \& Sons Inc., Canada, $3^{\mathrm{a}}$ ed., 307 p.

BROOKSHAW, D.R.; COKER, V.S.; LLOYD, J.R.; VAUGHAN, D.J.; PATTRICK, R.A.D. 2014. Redox interactions between $\mathrm{Cr}(\mathrm{VI})$ and $\mathrm{Fe}(\mathrm{II})$ in bioreduced biotite and chlorite. Environmental Science \& Technology, 48(19): 11337-11342. https:// doi.org/10.1021/es5031849

BROWN, P.A.; GILL, S.A.; ALLEN, S.J. 2000. Review paper - Metal removal from wastewater using peat. Water Research, 34(16): 3907-3916. https://doi.org/10.1016/ S0043-1354(00)00152-4

BUERGE, I.J.; HUG, S.J. 1997. Kinetics and $\mathrm{pH}$ dependence of chromium(VI) reduction by iron(II). Environmental Science \& Technology. 31(5): 1426-1432. https://doi. org/10.1021/es960672i

CALDER, L.M. 1988. Chromium contamination of groundwater. In: J.O. Nriagu \& E. Nieboer (ed.) Chromium in the natural and human environments. New York, Wiley, p. 215-230.

CAO, J.; ZHANG, W-H. 2006. Stabilization of chromium ore processing residue (COPR) with nanoscale iron particles. Journal of Hazardous Materials, 132(23): 213-219. https://doi.org/10.1016/j. jhazmat.2005.09.008

CERQUEIRA, S.C.A.; ROMÃO, L.P.C.; LUCAS, S.C.O.; FRAGA, L.E.; SIMÕES, 
M.L.; HAMMER, P.; LEAD, J.R.; MANGONI, A.P.; MANGRICH, A.S. 2012. Spectroscopic characterization of the reduction and removal of chromium(VI) by tropical peat and humin. Fuel, 91(1): 141-146.http://dx.doi.org/10.1016/j. fuel.2011.06.023

CETESB - COMPANHIA AMBIENTAL DE SÃO PAULO. 2012. Diretoria de Controle e Licenciamento Ambiental. Texto explicativo relação de áreas contaminadas e reabilitadas no Estado de São Paulo. Disponível em http://www.cetesb.sp.gov.br/userfiles/file/ areascontaminadas/2012/textoexplicativo.pdf. Acessado em 12 dez. 2014.

CETESB - COMPANHIA AMBIENTAL DE SÃO PAULO. 2013. Diretoria de Controle e Licenciamento Ambiental. Texto explicativo relação de áreas contaminadas e reabilitadas no Estado de São Paulo. Disponível em http://www.cetesb.sp.gov.br/userfiles/file/ areascontaminadas/2013/textoexplicativo. pdf. Acessado em 06 dez. 2013.

CETESB - COMPANHIA AMBIENTAL DE SÃO PAULO. 2016. Decisão de Diretoria $N^{\circ}$ 256/2016, de 22 de novembro de 2016. Diário oficial do Estado de São PauloCaderno Executivo I, 126 (219): 55-56.

\section{CETESB - COMPANHIAAMBIENTAL DE SÃO} PAULO. 2017. Crômio e seus compostos Ficha de informação toxicológica. https:// cetesb.sp.gov.br/laboratorios/wp-content/ uploads/sites/24/2019/06/Crômio.pdf

CHEN, C.; KUKKADAPU, R.; SPARKS, D.L. 2015. Influence of Coprecipitated Organic Matter on $\mathrm{Fe}^{2+}{ }_{(\text {aq) }}$ Catalyzed Transformation of Ferrihydrite: Implications for Carbon Dynamics. Environmental Science \& Technology, 49(18): 10927-10936. https:// doi.org/10.1021/acs.est.5b02448

CHEN, K.-Y.; TZOU, Y.-M.; CHAN, Y.-T.; WU, J.-J.; TEAH, H.-Y.; LIU, Y.-T. 2019. Removal and simultaneous reduction of $\mathrm{Cr}(\mathrm{VI})$ by organo-Fe(III) composites produced during coprecipitation and coagulation processes. Journal of Hazardous Materials, 376: 12-20. https:// doi.org/10.1016/j.jhazmat.2019.04.055
COCOZZA, C.; D'ORAZIO, V.; MIANO, T.T.; SHOTYK, W. 2003. Characterization of solid and aqueous phases of a peat bog profile using molecular fluorescence spectroscopy, ESR and FT-IR, and comparison with physical properties. Organic Geochemistry, 34: 49-60. https://doi.org/10.1016/S01466380(02)00208-5

CONAMA - CONSELHO NACIONAL DO MEIO AMBIENTAL. 2005. Resolução $n^{\circ}$ 357. Data da legislação: 17/03/2005 Publicação DOU de 18/03/2005, 25 p.

CONAMA - CONSELHO NACIONAL DO MEIO AMBIENTAL. 2011. Resolução $n^{\circ}$ 430. Data da legislação: 13/05/2011 Publicação DOU n ${ }^{\circ}$ 92, de 16/05/2011, 89 p.

DEIANA, S.; GESSA, C.; MANUNZA, B.; RAUSA, R.; SOLINAS, V. 1995. Iron(III) reduction by natural humic acids: a potentiometric and spectroscopic study. European Journal of Soil Science, 46: 103-108. https://doi. org/10.1111/j.1365-2389.1995.tb01816.x

EARY, L.E.; RAI, D. 1987. Kinetics of chromium(III) oxidation to chromium(VI) by reaction with manganese dioxide. Environmental Science \& Technology, 21(12): 1187-1193. https://doi.org/10.1021/ es00165a005

EARY, L.E.; RAI, D. 1988. Chromate removal from aqueous waste by reduction with ferrous iron. Environmental Science \& Technology, 22(7): 972-977. https://doi. org/10.1021/es00173a018

EARY, L.E.; RAI, D. 1989. Kinetics of chromate reduction by ferrous ions derived from hematite and biotite at $25^{\circ} \mathrm{C}$. American Journal of Science, 289: 180-213. https:// doi.org/10.2475/ajs.289.2.180

ECKERT, J.M.; STEWART, J.J.; WAITE, T.D.; SZYMCZAK, R.; WILLIAMS, K.L. 1990. Reduction of chromium(VI) at sub- $\mu \mathrm{g}^{-1}$ levels by fulvic acid. Analytica Chimica Acta, 236(1): 357-362. https://doi. org/10.1016/S0003-2670(00)83334-6 
ESSINGTON, M.E. 2005. Soil and water chemistry: An integrative approach. CRC Press, 534 p.

FENDORF, S.E. 1995. Surface reactions of chromium in soils and waters. Geoderma, 65: 55-71. https://doi.org/10.1016/00167061(94)00062-F

FENDORF, S.E.; ZASOSKI, R.J. 1992. Chromium(III) oxidation by $\delta-\mathrm{MnO}_{2}$, Characterization. Environmental Science \& Technology, 26(1): 79-85. https://doi. org/10.1021/es00025a006

FENDORF, S.E.; FENDORF, M.; SPARKS, D.L.; GRONSKY, R. 1992. Inhibitory mechanisms of $\mathrm{Cr}(\mathrm{III})$ oxidation by $\delta-\mathrm{MnO}_{2}$. Journal of Colloid and Interface Science, 153: 37-54. https://doi.org/10.1016/00219797(92)90296-X

FRANCHI, J.G.; SÍGOLO, J.B.; MOTTA, J.F.M. 2006. Diagnóstico das turfas no Brasil: histórico da utilização, classificação, geologia e dados econômicos. Revista Brasileira de Geociências, 36: 179190. https://doi.org/10.25249/03757536.200636S1179190

FRANCO, D.V. 2008. Tratamento redutivo de solo e água subterrânea contaminados com cromo hexavalente. Instituto de Química, Universidade Estadual de Campinas, Campinas, Tese de Doutorado, $183 \mathrm{f}$.

FUKUSHIMA, M.; NAKAYASU, K.; TANAKA, S.; NAKAMURA, H. 1995. Chromium(III) binding abilities of humic acids. Analytica Chimica Acta. 317: 195-206. https://doi. org/10.1016/0003-2670(95)00410-6

GOMES, M.A.C.; HAUSER-DAVIS, R.A.; SUZUKI, M.S.; VITÓRIA, A.P. 2017. Plant chromium uptake and transport, physiological effects and recent advances in molecular investigations. Ecotoxicology and Environmental Safety, 140: 55-64. https:// doi.org/10.1016/j.ecoenv.2017.01.042

GRÖHLICH, A.; LANGER, M.; MITRAKAS, M.; ZOUBOULIS, A.; KATSOYIANNIS, I.; ERNST, M. 2017. Effect of Organic Matter on $\mathrm{Cr}(\mathrm{VI})$ Removal from Groundwaters by $\mathrm{Fe}(\mathrm{II})$ Reductive Precipitation for
Groundwater Treatment. Water, 9(6): 389. https://doi.org/10.3390/w9060389

GUSTAFSSON, J.P.; ÉRSSPN, I.; OROMIEH, A.G.; van SCHAIK, J.W.J.; SJÖSTEDT, C.; KLEJA, D.B. 2014. Chromium(III) complexation to natural organic matter: mechanisms and modeling. Environmental Science \& Technology, 48: 1753-1761. https://doi.org/10.1021/es404557e

HAWLEY, E.L.; DEEB, R.A.; KAVANAUGH, M.C.; JACOBS, J.A. 2004. Treatment technologies for chromium(VI). In: J. Guertin, J.A. Jacobs, C.P. Avakian (eds.) Cr(VI) handbook. New York, CRC Press, chap. 8: 273-308. https://doi.org/10.1002/ chin. 200614270

HENRYK, K.; JAROSŁAW, C.; WITOLD, Ż. 2015. Peat and coconut fiber as biofilters for chromium adsorption from contaminated wastewaters. Environmental Science and Pollution Research, 23(1): 527-534. https:// doi.org/10.1007/s11356-015-5285-x

HORI, M.; SHOZUGAWA, K.; MATSUO, M. 2015. Reduction process of $\mathrm{Cr}(\mathrm{VI})$ by Fe(II) and humic acid analyzed using high time resolution XAFS analysis. Journal of Hazardous Materials, 285: 140-147. https:// doi.org/10.1016/j.jhazmat.2014.11.047

HU, Y.; ZHU, J.; LIU, Y. 2017. Removal of chromium(VI) from aqueous solutions by electrochemical reduction-precipitation. International Journal of Electrochemical Science, 12: 11387-11396. https://doi. org/10.20964/2017.12.12

HU, Y.; XUE, Q.; TANG, J.; FAN, X.; CHEN, H. 2019. New insights on $\mathrm{Cr}(\mathrm{VI})$ retention by ferrihydrite in the presence of $\mathrm{Fe}(\mathrm{II})$. Chemosphere, 222: 511-516. https://doi. org/10.1016/j.chemosphere.2019.01.160

HWANG, I.; BATCHELOR, B.; SCHLAUTMAN, M.A.; WANG, R. 2002. Effects of ferrous iron and molecular oxygen on chromium(VI) redox kinetics in the presence of aquifer solids. Journal of Hazardous Materials, 92: 143-159. https://doi.org/10.1016/S03043894(02)00006-7 
INDA Jr., A.V. 2002. Caracterização de goethita e hematita em solos poligenéticos. Universidade Federal do Rio Grande do Sul, Porto Alegre, Tese de Doutorado, 126 p.

INDA Jr., A.V.; KÄMPF, N. 2005. Variabilidade de goethita e hematita via dissolução redutiva em solos de região tropical e subtropical. Revista Brasileira de Ciência do Solo, 29:851-866. http://dx.doi.org/10.1590/ S0100-06832005000600003

JAMES，B.R; PETURA，J.C; VITALE， R.J.; MUSSOLINE, G.R. 1997. Oxidationreduction chemistry of chromium: Relevance to the regulation and remediation of chromate-contaminated soils, Journal of Soil Contamination, 6(6): 569-580. https:// doi.org/10.1080/15320389709383590

JIANG, W.; CAI, Q.; XU, W.; YANG, M.; CAI, Y.; DIONYSIOU, D.D.; O’SHEA, K.E. 2014. $\mathrm{Cr}(\mathrm{VI})$ Adsorption and reduction by humic acid coated on magnetite. Environmental Science \& Technology, 48(14): 8078-8085. https://doi.org/10.1021/es405804m

KASIULIENE, A.; CARABANTE, I.; BHATTACHARYA, P.; CAPORALE, A.G.; ADAMO, P.; KUMPIENE, J. 2018. Removal of metal(oid)s from contaminated water using iron-coated peat sorbent. Chemosphere, 198: 290-296. https://doi. org/10.1016/j.chemosphere.2018.01.139

KER, J.C. 1998. Latossolos do Brasil: uma revisão. Geonomos, 5: 17-40. https://doi. org/10.18285/geonomos.v5i1.187

KIM, C.; LAN, Y.; DENG, B. 2007. Kinetic study of hexavalent $\mathrm{Cr}(\mathrm{VI})$ reduction by hydrogen sulfide through goethite surface catalytic reaction. Geochemical Journal, 41(6): 397-405. https://doi.org/10.2343/ geochemj.41.397

KIM, J.G.; DIXON, J.B.; CHUSUEI, C.C; DENG, Y. 2002. Oxidation of chromium(III) to (VI) by manganese oxides. Soil Science Society of America Journal, 66: 306-315. https:// doi.org/10.2136/sssaj2002.0306

KIMBROUGH, D.E.; COHEN, Y.; WINER, A.M.; CREELMAN,L.;MABUNI,C.A.1999.Critical assessment of chromium in the environment.
Critical Reviews in Environmental Science and Technology, 29(1): 1-46. https://doi. org/10.1080/10643389991259164

KLEBER, M.; JOHNSON, M.G. 2010. Advances in understanding the molecular structure of soil organic matter: Implications for interactions in the environment. Advances in Agronomy, 106: 77-142. https://doi. org/10.1016/S0065-2113(10)06003-7

KÖGEL-KNABNER, I. 2017. The macromolecular organic composition of plant and microbial residues as inputs to soil organic matter: fourteen years on. Soil Biology and Biochemistry, 105: A3-A8. https://doi. org/10.1016/j.soilbio.2016.08.011

KWAK, S.; YOO, J.-C.; MOON, D.H.; BAEK, K. 2018. Role of clay minerals on reduction of Cr(VI). Geoderma, 312: 1-5. http://dx.doi. org/10.1016/j.geoderma.2017.10.001

MACHADO, J.M.C.; OLIVEIRA, L.M.C.P.E.; KAMOGAWA, M.Y. 2011. Reciclagem do crômio de resíduos químicos provenientes da determinação de carbono oxidável em fertilizantes orgânicos. Química Nova. 34(1): 131-134. http://dx.doi.org/10.1590/ S0100-40422011000100024

MAK, M.S.H.; LO, I.M.C. 2011. Influences of redox transformation, metal complexation and aggregation of fulvic acid and humic acid on $\mathrm{Cr}(\mathrm{VI})$ and $\mathrm{As}(\mathrm{V})$ removal by zero-valent iron. Chemosphere, 84(2): 234-240. http:// doi.org/10.1016/j.chemosphere.2011.04.024

MITRAKAS, M.G.; PANTAZATOU, A.S.; TZIMOU-TSITOURIDOU, R.; SIKALIDIS, C.A. 2011. Influence of $\mathrm{pH}$ and temperature on $\mathrm{Cr}(\mathrm{VI})$ removal from a natural water using $\mathrm{Fe}(\mathrm{II})$ : A pilot and full scale case study. Desalination and Water Treatment, 33(1-3): 77-85. https://doi.org/10.5004/ dwt.2011.2620

MOHAN, D.; PITTMAN Jr., C.U. 2006. Activated carbons and low cost adsorbents for remediation of tri- and hexavalent chromium from water. Journal of Hazardous Materials, 137(2): 762-811. https://doi. org/10.1016/j.jhazmat.2006.06.060 
MOORE, P.D. 1989. The ecology of peat-forming processes: a review. International Journal of Coal Geology, 12: 89-103.

MULLIGAN, C.N.; YONG, R.N.; GIBBS, B.F. 2001. Remediation technologies for metalcontaminated soils and groundwater: an evaluation. Engineering Geology, 60: 193-207. https://doi.org/10.1016/S0013-7952(00)00101-0

OLAZABAL, M. A.; NIKOLAIDIS, N. P.; SUIB, S. A.; MADARIAGA, J. M. 1997. Precipitation Equilibria of the Chromium (VI)/Iron(III) system and spectrospcopic characterizations of the precipitates. Environmental Science Technology, 31:28982902. https://doi.org/10.1021/es9700865

OTHA, A.; KAGI, H.; TSUNO, H.; NOMURA, M.; OKAI, T. 2012. Speciation study of $\mathrm{Cr}(\mathrm{VI} / \mathrm{III})$ reacting with humic substances and determination of local structure of $\mathrm{Cr}$ binding humic substances using XAFS spectroscopy. Geochemical Journal, 46: 409420. https://doi.org/10.2343/geochemj.2.0222

OWLAD, M.; AROUA, M.K.; DAUD, W.A.W.; BAROUTIAN, S. 2008. Removal of Hexavalent Chromium-Contaminated Water and Wastewater: A Review. Water, Air, and Soil Pollution, 200(1-4): 59-77. http://doi. org/10.1007/s11270-008-9893-7

PAGE, S.E.; RIELEY J.O.; WÜST, R. 2006. Lowland tropical peatlands of Southeast Asia. In: I.P. Martini, A.M. Cortizas, W. Chesworth (eds.) Peatlands: evolution and records of environmental and climate changes. Amsterdam, Elsevier, p.145-172. https://doi. org/10.1016/S0928-2025(06)09007-9

PALMER, C.D.; PULS, R.W. 1994. Natural attenuation of hexavalent chromium in groundwater and soils. EPA Ground Water Issue, U.S.EPA/540/S-94/505.

PALMER, C.D.; WITTBRODT, P.R. 1991. Processes affecting the remediation of chromium contaminated sites. Environmental Health Perspectives, 92(1): 25-40. http://doi.org/10.1289/ehp.919225

PARK, S.-J.; JANG, Y.-S. 2002. Pore structure and surface properties of chemically modified activated carbons for adsorption mechanism and rate of $\mathrm{Cr}(\mathrm{VI})$. Journal of Colloid and Interface Science, 249(2): 458-463. https:// doi.org/10.1006/jcis.2002.8269

PUGAS, M.S. 2007. Íons de metais pesados Ni, $\mathrm{Cu}$ e $\mathrm{Cr}$ em área impactada por resíduo de galvanoplastia na região metropolitana de São Paulo - SP. Instituto de Geociências, Universidade de São Paulo, São Paulo, Dissertação de Mestrado, 82 p. https://doi. org/10.11606/D.44.2007.tde-02052007114138

RAI, D.; EARY, L.E.; ZACARA, J.M. 1989. Environmental chemistry of chromium. The Science of the Total Environment, 86(12): 15-23. https://doi.org/10.1016/00489697(89)90189-7

RICHARD, F.C.; BOURG,A.C.M. 1991.Aqueous geochemistry of chromium: a review. Water Research, 25(7): 807-816. https://doi. org/10.1016/0043-1354(91)90160-R

RIZZUTI, A.M.; NEWKIRK, C.R.; WILSON, K.A.; COSME, L.W.; COHEN, A.D. 2017. Biosorption of hexavalente chromium from aqueous solutions using highly characterised peats. Mires and Peat, 19: 1-10. https://doi. org/10.19189/MaP.2016.OMB.248

SAMPAIO, J.A.; ANDRADE, M.C; PAIVA, P.R.P. 2008. Cromita. In: A.B. Luz \& F.A.F. Lins (eds.) Rochas e minerais industriais: Uso e especificações. Rio de Janeiro, CETEM/MCT, $2^{\text {a }}$ ed., cap. 18: 403-426.

SANTOS, A.M.P.; BERTOLI, A.C.; BORGES, C.P.; GOMES, R.A.B.G.; GARCIA, J.S.; TREVISAN, M.G. 2018. New organomineral complex from humic substances extracted from poultry wastes: Synthesis, characterization and controlled release study. Journal of the Brazilian Chemical Society, 29(1): 140-150. http:// dx.doi.org/10.21577/0103-5053.20170122

SANTOS, M.M.A.; SHINZATO, M.C.; FREITAS, J.G. 2018. Characterization and evaluation of a tropical peat for the removal of $\mathrm{Cr}(\mathrm{VI})$ from solution. Environmental Earth Sciences, 77(13): 515. https://doi. org/10.1007/s12665-018-7703-0 
SCHLAUTMAN, M.A.; HAN, I. 2001. Effects of $\mathrm{pH}$ and dissolved oxygen on the reduction of hexavalente chromium by dissolved ferrous iron in poorly buffered aqueous systems. Water Resource, 35(6): 1534-1546. https:// doi.org/10.1016/S0043-1354(00)00408-5

SHAHID, M.; SHAMSHAD, S.; RAFIQ, M.; KHALID, S.; BIBI, I.; NIAZI, N.K.; DUMAT, C.; RASHID, M.I. 2017. Chromium speciation, bioavailability, uptake, toxicity and detoxification in soilplant system: A review. Chemosphere, 178: 513-533. https://doi.org/10.1016/j. chemosphere.2017.03.074

SKOOG, D.A.; WEST, D.M.; HOLLER, J.F.; CROUCH, S.R. 2004. Fundamentals of analytical chemistry. Thomson Brooks/ Cole, Belmont, $8^{\circ}$ ed., 992 p.

SODANO, M.; LERDA, C.; NISTICÒ, R.; MARTIN, M.; MAGNACCA, G.; CELI，L.; SAID-PULLICINO，D. 2017. Dissolved organic carbon retention by coprecipitation during the oxidation of ferrous iron. Geoderma, 307: 19-29. https:// doi.org/10.1016/j.geoderma.2017.07.022

SPARKS, D.L. 2003. Environmental soil chemistry. Academic Press, Elsevier Science, $2^{\circ}$ ed., $352 \mathrm{p}$.

SPOSITO, G. 2008. The chemistry of soils. Oxford University Press, New York, $2^{\circ}$ ed., $329 \mathrm{p}$.

SREERAM, K.J.; RAMASIMI, T. 2001. Speciation and recovery of chromium from chromite ore processing residues. Journal of Environmental Monitoring, 3(5): 526-530. https://doi.org/10.1039/b104303j

STANIN, F.T.; PIRNIE, M. 2004. The transport and fate of chromium(VI) in the environment. In: J. Guertin, J.A Jacobs, C.P. Avakian (eds.) Cr(VI) Handbook. New York, CRC Press, chap. 5: 161-211. https:// doi.org/10.1201/9780203487969

TADINI, A.M.; MOREIRA, A.B.; BISINOTI, M.C. 2014. Fractionation of aquatic humic substances and dynamic of chromium species in an aquatic body influenced by sugarcane cultivation. Journal of the Brazilian Chemical
Society, 25(1): 119-125. http://dx.doi. org/10.5935/0103-5053.20130277

TOMBÁCZ, E.; LIBOR, Z.; ILLÉS, E.; MAJZIK, A.; KLUMPP, E. 2004. The role of reactive surface sites and complexation by humic acids in the interaction of clay mineral and iron oxide particles. Organic Geochemistry, 35(3): 257-267. https://doi.org/10.1016/j. orggeochem.2003.11.002

TREMOCOLDI, W. A. 2003. A mineralogia dos silicatos e dos óxidos de ferro da fração argila de solos desenvolvidos de rochas básicas no estado de São Paulo. Revista Biociências, 9(1): 15-22.

USEPA - UNITED STATES ENVIRONMENT PROTECTION AGENCY. 2017. Chromium in drinking water. Disponível em https:// www.epa.gov/dwstandardsregulations/ chromium-drinking-water. Acessado em 10 set. 2018 .

USMAN, M.; BYRNE, J.M.; CHAUDHARY, A.; ORSETTI, S.; HANNA, K.; RUBY, C.; KAPPLER, A.; HADERLEIN, S.B. 2018. Magnetite and green rust: Synthesis, properties, and environmental applications of mixed-valent iron minerals. Chemical Reviews, 118(7): 3251-3304. https://doi. org/10.1021/acs.chemrev.7b00224

VINUTH, M.; NAIK, H.S.B.; SEKHAR, K.C.; MANJANNA, J.; VINODA, B.M. 2015. Environmental remediation of hexavalent chromium in aqueous medium using fe(ii)-montmorillonite as reductant. Procedia Earth and Planetary Science, 11: 275-283. https://doi.org/10.1016/j. proeps.2015.06.036

WANG, L.P.; ZHANG, M.Y.; HUANG, Z.C.; LONG, X.P. 2016. Adsorption behavior of $\mathrm{ACF} / \mathrm{CNT}$ composites for $\mathrm{Cr}(\mathrm{VI})$ from aqueous solution. In: P. Chen (ed). Material Science and Environmental Engineering. London, Taylor \& Francis Group, $3^{\text {rd }}$ ed., p. 207-214.

WITTBRODT, P.R.; PALMER, C.D. 1996. Effect of temperature, ionic strength, background electrolytes and $\mathrm{Fe}(\mathrm{III})$ on the reduction of hexavalente chromium by soil humic substances, Environmental Science \& 
Technology, 30(8): 2470-2477. https://doi. org/10.1021/es950731c

XIAO, W.; ZHANG, Y.; LI, T.; CHEN, B.; WANG, H.; HE, Z.; YANG, X. 2012. Reduction kinetics of hexavalent chromium in soils and its correlation with soil properties. Journal of Environment Quality, 41(5): 1452-1458. https://doi.org/10.2134/jeq2012.0061

YE, C.; BAI, T.; YANG, Y.; ZHANG, H.; GUO, H.; LI, Z.; LI, H; HU, S. 2017. Physical access for residue-mineral interactions controls organic carbon retention in an Oxisol soil. Scientific Reports, 7(1): 1-9. https://doi.org/10.1038/s41598-017-06654-6

ZACHARA, J.M.; COWAN, C.E.; SCHMIDT,
R.L.; AINSWORTH, C.C. 1988. Chromate adsorption by kaolinite. Clays and Clays Minerals, 36(4): 317-326.

ZHANG, J.; YIN, H.; BARNIE, S.; WEI, M.; CHEN, H. 2019. Mechanism and modeling of hexavalent chromium interaction with a typical black soil: the importance of the relationship between adsorption and reduction. Royal Society of Chemistry (RSC Advances), 9: 5582-5591. https://doi. org/10.1039/C8RA08154A

ZHILIN, D.M.; SCHMITT-KOPPLIN, P.; PERMINOVA, I.V. 2004. Reduction of $\mathrm{Cr}(\mathrm{VI})$ by peat and coal humic substances. Environmental Chemical Letter, 2(3): 141-145. https://doi.org/10.1007/s10311-004-0085-4

\section{Endereço dos autores:}

Vanessa Maronezi, Marília Mayumi Augusto dos Santos, Danielle Bittencourt Faria, Maria Isabel Garcia Rosa, Mirian Chieko Shinzato - Instituto de Ciências Ambientais, Químicas e Farmacêuticas, Universidade Federal de São Paulo (UNIFESP), Rua São Nicolau, 210, CEP 09913-030, Diadema, SP. E-mails: ea.vmaronezi@gmail.com; mayumimarilia@gmail.com; daniielle.bitencourt@gmail.com; misabelgrosa@gmail.com; mirian.chieko@unifesp.br

Artigo submetido em 10 de julho de 2019, aceito em 20 de agosto de 2019. 